\title{
Deficits on self ordered tasks associated with hyperostosis frontalis interna
}

\author{
Greig I de Zubicaray, Jonathan B Chalk, Stephen E Rose, James Semple, Glen A Smith
}

Centre for Magnetic Resonance

G I de Zubicaray

S E Rose

\section{Department of Psychology, \\ The University of \\ Queensland 4072, \\ Australia \\ G A Smith}

Department of

Medicine,

The University of

Queensland, Princess

Alexandra Hospital,

Woolloongabba 4102,

Australia

J B Chalk

SmithKline Beecham

Pharmaceuticals,

Clinical Pharmacology

Unit, New Frontiers

Science Park, Third

Avenue, Harlow, Essex

CM19 5AW, UK

J Semple

Correspondence to: Dr Jonathan B Chalk, Department of Medicine, The University of Queensland, Princess Alexandra Hospital, Woolloongabba 4102 , Australia.

Received 2 January 1997 and in revised form 2 April 1997 Accepted 4 April 1997

\begin{abstract}
A 74 year old patient, EW, with dorsolateral frontal cortical compression due to hyperostosis frontalis interna, in the absence of the Morgagni or Stewart-Morel syndromes, is described. In addition to conventional neuropsychological measures EW was administered one nonspatial and two spatial self ordered working memory tasks, as well as a standard measure of fluid intelligence or $\mathrm{g}$. She showed impaired performance on all three self ordered working memory tasks compared with a normal control group of 10 subjects matched for age, education, sex, and IQ. By contrast, her performance on the fluid intelligence test was comparable with that of the controls. It is concluded that the compression of dorsolateral frontal cortex accompanying hyperostosis frontalis interna may produce selective cognitive impairment.
\end{abstract}

(F Neurol Neurosurg Psychiatry 1997;63:309-314)

Keywords: hyperostosis frontalis interna; self ordered tasks; working memory

Hyperostosis frontalis interna (HFI) is a disorder involving progressive symmetric thickening of the inner table of the frontal bone of the human skull. It is predominantly found in older women, and is thought to be the result of a more generalised disorder of bone metabolism. ${ }^{1}$ The condition was first described by Giovanni Batistta Morgagni, ${ }^{2}$ who noted an association between thickening of the frontal bone and both obesity and hirsutism. Stewart and Morel later documented neuropsychiatric sequelae in some cases, ${ }^{34}$ leading to the use of the labels Morgagni syndrome or StewartMorel syndrome.

Although HFI has been recognised for over 250 years, the neurological and neuropsychological implications of the disorder remain vague. ${ }^{5}$ In addition, it has been suggested that the disorder has no pathological relevance. ${ }^{6}$ Descriptions of the disorder are consequently found in only a handful of textbooks. Aside from the increased ossification of the frontal bone, an associated feature is compression of the immediately adjacent tissue of the frontal cortex, a region responsible for diverse cognitive functions. Two recent neuropsychological formulations draw attention to the part that this region plays in self ordered working memory, ${ }^{78}$ and in fluid intelligence or novel problem solving. ${ }^{9} 10$
According to Petrides, ${ }^{8}$ the mid-dorsolateral frontal cortex is involved in the monitoring of self generated and externally generated responses. In a typical self ordered task the subject is presented with a set of stimuli and is required to select each stimulus only once. Therefore, the task requires the subject to monitor his or her performance within working memory. Externally ordered tasks require the subject to monitor those items from a set of stimuli that have already been selected and those items that have not been selected. The number of possible alternatives that need to be considered in working memory is posited to be the "critical factor" in determining whether a deficit will be produced after a lesion to the mid-dorsolateral frontal cortex. ${ }^{8}$ Evidence from lesion studies ${ }^{11} 12$ and PET studies of humans supports a role for this cortical region in the performance of these tasks. ${ }^{7}$

Duncan has recently proposed that fluid intelligence or novel problem solving may be largely a reflection of frontal cortical functions. ${ }^{9}$ According to Duncan, ${ }^{9}$ the widespread disorganisation of behaviour seen after frontal lobe lesions is reminiscent of the concept of "general intelligence" or Spearman's "g". ${ }^{13}$ Although conventional tests of psychometric intelligence such as the Wechsler adult intelligence scale (WAIS) have been considered unsuitable for disclosing deficits associated with frontal lobe lesions, ${ }^{14}{ }^{15}$ tests of fluid intelligence may be particularly appropriate for assessing frontal lobe dysfunction due to their strong correlations with $\mathrm{g}$, and their emphasis on novel problem solving. In a recent investigation, patients with frontal cortical lesions and superior IQs as assessed by the WAIS showed impairments of $20-60$ points on a standard measure of fluid intelligence. ${ }^{10}$

The present study was designed to investigate the neuropsychological sequelae of the frontal cortical compression associated with HFI. In this paper we describe a subject who was found to have HFI on MRI, in the absence of complications associated with the Morgagni or Stewart-Morel syndromes (obesity, hirsutism, and neuropsychiatric symptoms). Her performance on a set of standard neuropsychological tests and on several experimental measures designed to assess hypotheses from the two theories of frontal lobe functioning outlined above, is discussed.

\section{Case description}

EW, a 74 year old right handed woman volunteered as a normal control subject for a longitudinal investigation of Alzheimer's 


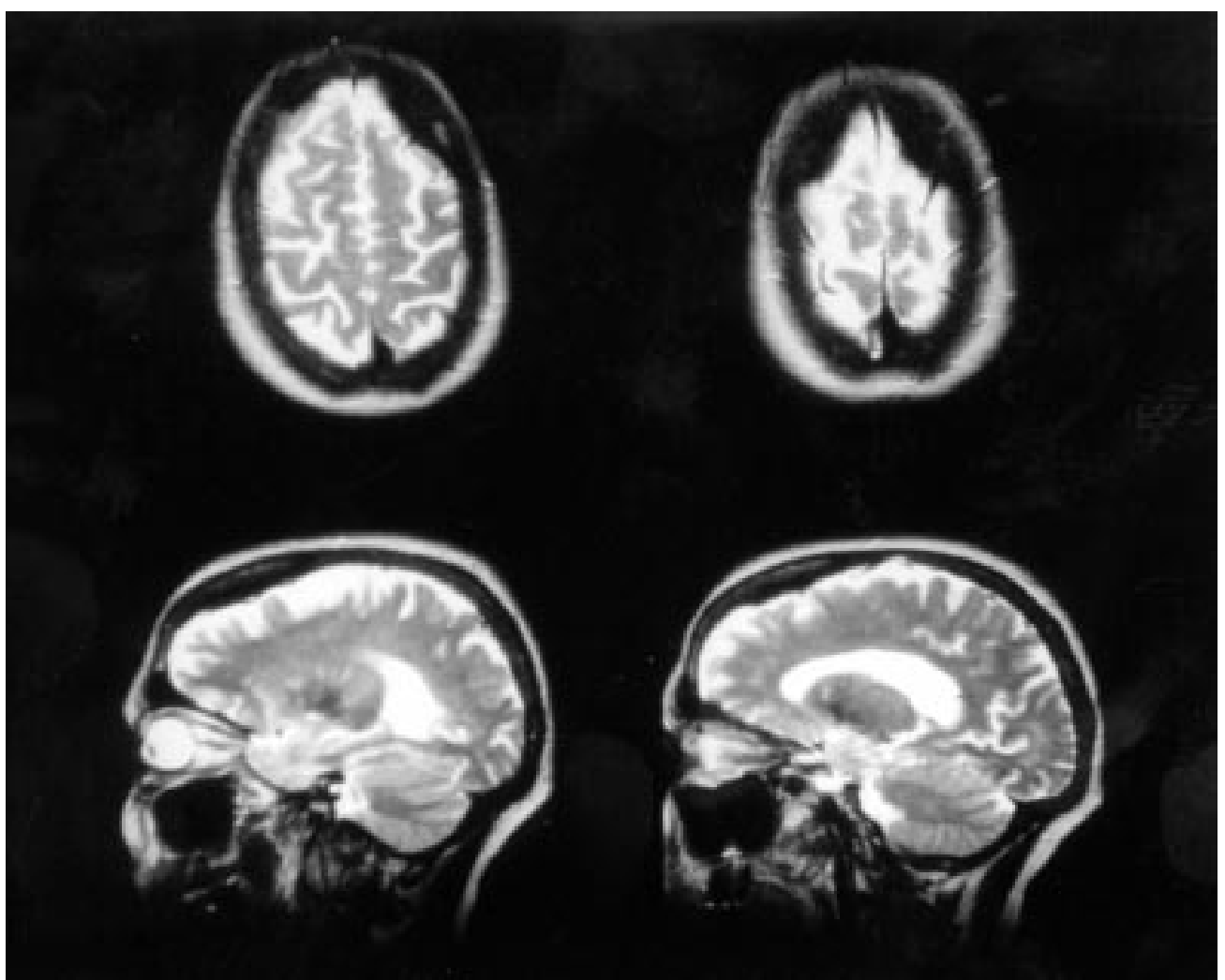

Figure 1 Two T2 weighted sagittal (bottom) and axial (top) images of the frontal lobe region showing predominantly left sided compression of dorsolateral frontal cortex.

disease and normal aging conducted at the Centre for Magnetic Resonance, University of Queensland. She is about $157 \mathrm{~cm}$ tall and weighs $59 \mathrm{~kg}$. She has no history of head injury or neurological or psychiatric disorder. Brain MRI conducted on admission to the study disclosed her HFI. EW was born in the United Kingdom and attended school until she was about 14 years of age. EW then completed a three year registered nursing course. She emigrated to Australia in 1959, where she continued nursing in various hospitals until retiring at the age of 64 .

Little is known about the precise onset of her HFI. However, she recalls having experienced some difficulty organising and keeping track of patient details and records in the 12 months before her ceasing employment. As a result, she retired from her position a year early of her own volition. At interview, she described having difficulties with organising some everyday activities, which she attributed to her "rushing ahead" of herself. EW also reported occasionally performing activities in the wrong sequence. Detailed neurological examination was normal except for a positive palmomental reflex on the left side and an area of absent vibration sense on the left foot below the ankle.

\section{IMAGING STUDIES}

Hyperostosis of the frontal bones was clearly seen on computed axial tomography (not shown). Her brain was subsequently imaged using a Bruker $2 \mathrm{~T}$ whole body imaging system. Sixteen contiguous $5 \mathrm{~mm}$ T2 weighted axial and sagittal images of the brain were acquired using a rapid acquisition with relaxation enhancement (RARE) sequence. The imaging indices were TR 3s, effective TE $80 \mathrm{~ms}$, and a rare factor of 8 . The T2 weighted sagittal images and axial images show significant and predominantly left sided compression of dorsolateral frontal cortex (fig 1).

CLINICAL NEUROPSYCHOLOGICAL INVESTIGATIONS Table 1 shows the results of standard clinical neuropsychological tests administered on admission. On the mini mental state examination (MMSE) EW obtained a score of $28 .{ }^{16} \mathrm{Her}$ full scale IQ on the revised Wechsler adult intelligence scale (WAIS-R) was in the above average range, and consistent with her premorbid IQ estimated via the national adult reading test (NART). ${ }^{17}{ }^{18}$ The difference between her verbal and performance IQs, although significant, was of a magnitude found in $13 \%$ of the WAIS-R standardisation sample. Her performance on memory measures was well within the normal range. Measures of forward and backward digit span, and a measure of spatial span similar to the Corsi block tapping task taken from the Cambridge neuropsychological test automated battery (CANTAB), ${ }^{11}$ were performed within the normal range for her age group. On the recognition memory test she achieved a near perfect score for both words and faces. ${ }^{19}$ Her performance on the Hopkins verbal learning test (HVLT) was also above average. $^{20}$

EW also performed normally on tests of language ability, such as the Boston naming test, ${ }^{21}$ and the spot the word task from the speed and 
Table 1 Patient's performance on standard neuropsychological assessment

\begin{tabular}{|c|c|}
\hline Assessment & Score \\
\hline \multicolumn{2}{|l|}{ WAIS-R subtests (age scaled scores): } \\
\hline Information & 13 \\
\hline Digit span & 9 \\
\hline Vocabulary & 13 \\
\hline Arithmetic & 9 \\
\hline Comprehension & 10 \\
\hline Similarities & 10 \\
\hline Picture completion & 13 \\
\hline Picture arrangement & 16 \\
\hline Block design & 13 \\
\hline Object assembly & 14 \\
\hline Digit symbol & 10 \\
\hline Verbal IQ & 103 \\
\hline Performance IQ & 120 \\
\hline Full scale IQ & 111 \\
\hline Estimated premorbid IQ (NART) & 115 \\
\hline Mini mental state examination & $28 / 30$ \\
\hline \multicolumn{2}{|l|}{ Language: } \\
\hline Boston naming test & $55 / 60$ \\
\hline \multicolumn{2}{|l|}{ Speed and capacity of language processing: } \\
\hline Spot the word A (scaled score) & 16 \\
\hline Speed of comprehension A (scaled score) & 8 \\
\hline Discrepancy & $8(<5 \text { th \%ile })^{\star}$ \\
\hline \multicolumn{2}{|l|}{ Memory: } \\
\hline Forward digit span & 6 \\
\hline Backward digit span & 5 \\
\hline Spatial span & 5 (controls $4.9(\mathrm{SD} 0.6))$ \\
\hline \multicolumn{2}{|l|}{ Recognition memory test: } \\
\hline Words & $50 / 50$ \\
\hline Faces & $49 / 50$ \\
\hline \multicolumn{2}{|l|}{ Hopkins verbal learning test: } \\
\hline Free recall & $21 / 36$ \\
\hline Discrimination index & $11 / 12$ \\
\hline \multicolumn{2}{|l|}{ Visual perception: } \\
\hline \multicolumn{2}{|l|}{ Visual object and space perception battery: } \\
\hline Incomplete letters & 19 \\
\hline Silhouettes & 23 \\
\hline Object decision & 17 \\
\hline Progressive silhouettes & 8 \\
\hline Dot counting & $6(<5$ th \%ile $) \dagger$ \\
\hline Position discrimination & 19 \\
\hline Number location & 10 \\
\hline Cube analysis & 10 \\
\hline \multicolumn{2}{|l|}{ Executive: } \\
\hline Tower of London (average number of moves) & $2 / 2,3 / 3,5.25 / 4,5.5 / 5$ \\
\hline Verbal fluency (FAS, 60 seconds each) & 49 \\
\hline \multicolumn{2}{|l|}{ Six elements test: } \\
\hline Number of tasks attempted & $5 / 6$ \\
\hline Number of rule breaks & 0 \\
\hline Mean time on task & $2 \mathrm{~min}, 22 \mathrm{~s}$ \\
\hline Cognitive estimations & 7 errors \\
\hline \multicolumn{2}{|l|}{ Modified card sorting test: } \\
\hline Categories achieved & $3($ controls $4.6(\mathrm{SD} 1.1))$ \\
\hline Perseverative errors & 11 (controls 4.6 (SD 3.7)) \\
\hline
\end{tabular}

$\star$ Baddeley et $\mathrm{l}^{22}$; † Warrington and James. ${ }^{23}$

capacity of language processing (SCOLP) test. ${ }^{22}$ However, there was a significant discrepancy between her scaled scores on the spot the word and speed of comprehension subtests, indicating a relative impairment in semantic processing speed. Her performance on the visuoperceptual tests from the visual object and space perception battery (VOSP) was normal, ${ }^{23}$ except for the dot counting subtest that assesses single point localisation and visual scanning abilities, perhaps indicating some impairment in the function of the frontal eye fields. ${ }^{24} 25$

Standard measures of executive functioning disclosed variable performance. On the Tower of London task, ${ }^{1126}$ six elements test, ${ }^{27}{ }^{28}$ and measures of verbal fluency she performed in the normal range. Although her overall performance on the cognitive estimations test was normal, ${ }^{29} \mathrm{EW}$ did provide a "very extreme" estimate of the height of the Brisbane City Hall clock tower, asserting that it was 1000 yards tall (actual height 101 yards). She also showed borderline impairment on the modified card sorting test, ${ }^{30}$ a measure of category formation and mental flexibility.

In summary, she showed normal intellectual, memory, and language functioning with some evidence of moderate impairment in semantic processing speed and visual scanning, and borderline impairment in mental flexibility.

EXPERIMENTAL INVESTIGATIONS

As well as undergoing standard neuropsychological assessment, EW also completed several experimental measures designed to test her self ordered working memory and fluid intelligence. Her performance on these tasks was evaluated against that of 10 normal controls matched for age, education, gender, and full scale IQ (mean age 73.75 (SD 1.83) years; mean education 12.63 (SD 3.07) years; mean WAIS-R full scale IQ 114.88 (SD 4.12)). Controls were selected from a larger sample of normal older adults who were participating in the longitudinal study of Alzheimer's disease and normal aging at the Centre for Magnetic Resonance. All controls had undergone neurological examination as well as MRI scans.

SELF ORDERED COGNITION

Three tasks (two spatial, one non-spatial) were chosen:

\section{Self ordered pointing}

Petrides and Milner's self ordered pointing task requires the subject to organise a sequence of pointing responses to series of six, eight, 10, and 12 representational pictures (living and man made objects). ${ }^{31}$ Three consecutive trials are given at each level, and the subject is instructed not to touch any one stimulus more than once within a trial.

\section{Selective letter generation}

This non-spatial self ordered task, similar to Wiegersma, van der Scheer, and Human's task, ${ }^{32}$ requires the subject to selectively generate letters from the English alphabet within a 60 second period. Production is restricted to a different set of six letters for each of four trials. The subject is instructed to produce as many different letters as possible, with the provisos that he or she may not produce letters in alphabetical order either forward or backward, or repeat or alternate letters. Each set is printed in 42 point Times New Roman font on a card that is placed in front of the subject before commencing, and remains in view throughout the trial of that particular set. The following sets of letters were used: B to $\mathrm{G}, \mathrm{H}$ to $\mathrm{M}, \mathrm{N}$ to $\mathrm{S}$, and $\mathrm{T}$ to $\mathrm{Y}$.

\section{Spatial working memory}

This task from CANTAB requires the subject to search through boxes presented on a computer screen by touching them and disclosing their contents. ${ }^{11}$ The object is to collect a series of blue tokens from the boxes, and fill a column at the right hand corner of the screen. The instruction is that a blue token, once found in a box, will never be found in that particular box again. The number of tokens to be found corresponds to the number of boxes on the 


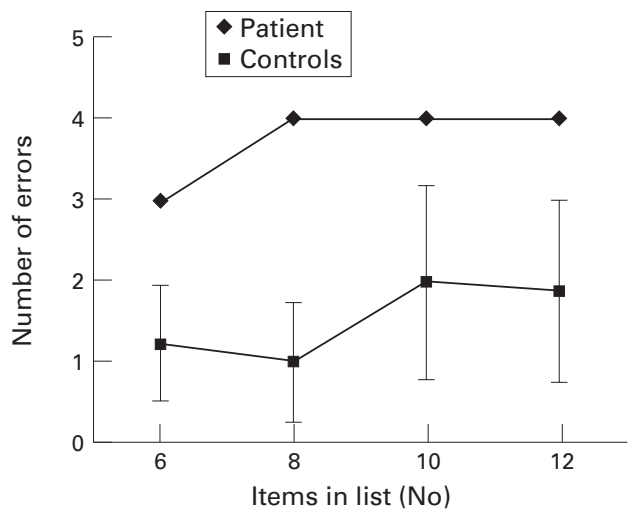

Figure $2 E W$ and the control group's total error scores for all four list lengths of the self ordered pointing task. Bars represent $S D$.

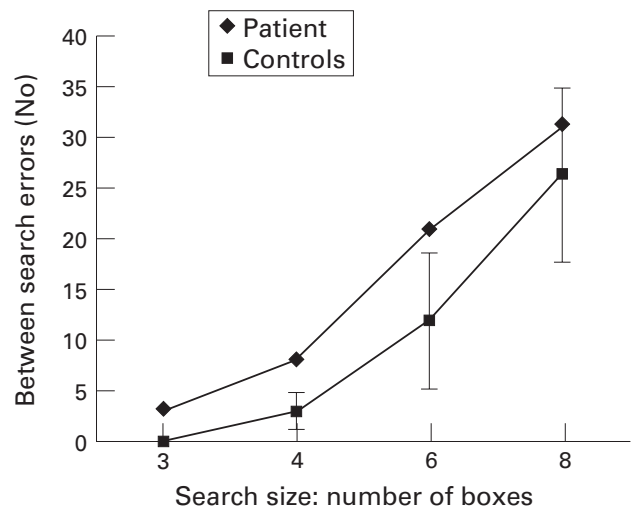

Figure $3 E W$ and the control group's between search errors on the spatial working memory task. Bars represent $S D$.

screen. Four trials each of three, four, six, and eight boxes were used. The task differs from the Petrides and Milner task in that the subject is not required to remember objects by their specific features, only by their respective locations.

\section{Results}

SELF ORDERED POINTING

Figure 2 shows the results for the self ordered pointing task. Her total error score of 15 was significantly poorer than that of the control group (mean 6.12 (SD 2.59)), giving a $\mathrm{z}$ score of 3.43. However, her performance tended to approach that of the controls as the number of items in each list increased, due to the greater variability in the controls' performance.

\section{SELECTIVE LETTER GENERATION}

The patient produced a total of 81 letters, and committed a total of 50 errors across the four trials. Conversely, the controls produced a mean (SD) total of 106.38 (20.1) letters, and committed a mean of $23.62(11.22)$ total

Table 2 WAIS-R and culture fair IQs of EW and controls

\begin{tabular}{llll}
\hline & WAIS-R IQ & Culture fair IQ & $\begin{array}{l}\text { WAIS-R-culture fair } \\
\text { IQ discrepancy }\end{array}$ \\
\hline EW & 111 & 87 & 24 \\
Controls $(\mathrm{n}=10)($ mean (SD)) & $114.8(4.12)$ & $82.38(12.39)$ & $32.5(11.4)$ \\
\hline
\end{tabular}

errors. Her total error score was significantly poorer than that of the controls ( $\mathrm{z}$ score of 2.35).

SPATIAL WORKING MEMORY

Figure 3 shows the patient's "between search" errors on the spatial working memory task. A between search error occurs when a subject returns to a box in which a blue counter has already been found. She made a total of 63 between search errors (controls' mean 40.88 (SD 14.16)), which was $1.56 \mathrm{SD}$ below the mean of the controls, giving a $\mathrm{z}$ score of 1.56 . However, she performed significantly poorer than the controls on the first two trials presented (three and four boxes), with $\mathrm{z}$ scores of 3 and 2.75 respectively. Her performance approximated to that of the controls on the last two trials (six and eight boxes), again due to the greater variability in their performance. A strategy measure was also calculated based on the number of different boxes used to initiate search sequences for the six and eight box trials. ${ }^{11}$ She achieved a strategy score of 38, which was not significantly different to the controls' mean score of 35.87 (SD 3.48).

FLUID INTELLIGENCE

Scale 2, form A of the Cattell culture fair intelligence test was administered individually to the patient and the 10 controls by the method of Duncan et al. ${ }^{10}{ }^{33}$ The culture fair test, a measure of fluid g, requires the subject to complete four subtests of novel problems: series, classification, matrices, and conditions (or topology), each within a specified time limit. Like the WAIS-R, the culture fair test provides IQs with a mean of 100 . However, unlike the WAIS-R's norms, which have an SD of 15, culture fair IQs have an SD of 16.

\section{Results}

Table 2 shows the patient's and the control group's scores on the culture fair test. Her culture fair IQ and culture fair IQ-WAIS-R full scale IQ discrepancy did not differ significantly from that of the controls ( $\mathrm{z}$ scores of 0.37 and 0.75 respectively).

\section{Discussion}

We have described a patient with selective deficits in self ordered working memory associated with the compression of the dorsolateral frontal cortex resulting from HFI. Despite having intact visual and verbal short term memory, and normal performance on several standard measures of executive functioning, EW had considerable difficulty with tasks requiring the active manipulation and monitoring of information in spatial and non-spatial working memory. This occurred in the absence of the Morgagni and Stewart-Morel syndromes, or any overt signs of behavioural impairment. By contrast with her apparent deficits on self ordered tasks, EW did not show an impairment on a standard measure of fluid intelligence or novel problem solving.

EW's deficits in self ordered working memory were more pronounced on the self ordered pointing and selective letter generation 
tasks than on the spatial working memory measure. This discrepancy may be attributable to different neuroanatomical substrates for performance of these tasks. Petrides and Milner found that patients with left frontal cortical lesions were more impaired on the self ordered pointing task than patients with right frontal lesions. ${ }^{31}$ In as much as the selective letter generation task employed here is a novel one, it may reasonably be assumed to be a verbal working memory task, and hence more dependent on functions mediated by the left hemisphere. The neuroanatomical substrate for performance of the spatial working memory task is less clearly lateralised. A recent PET study disclosed activation in the right middorsolateral frontal cortex associated with performance on this task, ${ }^{7}$ whereas two studies of patients with frontal lobe excisions failed to find differences in performance related to side of lesion. ${ }^{11}{ }^{12} \mathrm{EW}$ 's frontal cortical compression is predominantly left sided.

Although deficits in self ordered working memory have been reported in the past after frontal lobe lesions, ${ }^{11} 123132$ the patient's performance raises some questions regarding the "critical factor" in performance on these tasks. Petrides has suggested that the impairment on spatial working memory tasks that follows mid-dorsolateral frontal lesions depends on the number of alternative stimuli to be monitored. ${ }^{8}$ However, unlike the controls, who showed greater variability in their performance as the number of items in each set increased, EW tended to show greater impairment on the initial trials of the self ordered pointing and spatial working memory tasks that utilised sets comprising fewer items. A similar result has recently been reported on the spatial working memory measure for frontal lobe lesion patients. ${ }^{12}$ This present finding may represent an effect of task novelty, whereby a subject becomes more proficient at a task with successive trials. The importance of novelty has often been emphasised in models of executive processes, ${ }^{93435}$ and certainly no routine or rote method of searching is available to the subject on commencing the self ordered tasks.

The absence of impairment on the test of fluid intelligence may be due to various reasons. Firstly, it may be that only patients with superior IQs as assessed by conventional intelligence tests such as the WAIS-R will show significant impairment on the corresponding tests of fluid intelligence. ${ }^{10}$ However, several cases have been reported of patients with extensive frontal lesions and superior WAIS IQs who performed comparably on other standard fluid intelligence measures. ${ }^{28}{ }^{29} \mathrm{Sec}-$ ondly, g may represent the joint operation of certain functionally distinct regions within the frontal cortex, or the function of a relatively specific region of frontal cortex..$^{10}$ The patient's cortical compression is, to the best of our knowledge, limited to the dorsolateral frontal cortex. Thirdly, the slow progression of the hyperostosis and accompanying cortical compression that are the principal features of HFI may allow individual patients to develop compensatory processes that preclude the appear- ance of any sudden, pronounced impairment. The patient did not present with a widespread disorganisation of behaviour reminiscent of a dysexecutive syndrome. ${ }^{36}$

Little is known about the mechanisms involved in HFI, and those case reports that have been published have focused almost exclusively on the Morgagni or Stewart-Morel syndromes, which are not invariably found in association with HFI. At present, the evidence suggests that HFI is the result of a more generalised disorder of bone metabolism. ${ }^{1}$ It is most certainly considered a comparatively gender specific disorder of aging, occurring most often in older women. ${ }^{5}$ However, the neurological and neuropsychological sequelae of the disorder have not hitherto been subject to rigorous investigation.

The discovery of relatively selective cognitive deficits associated with the compression of the dorsolateral frontal cortex resulting from HFI raises some interesting questions. One question is why some authorities consider it to be of no pathological relevance when there have been so few detailed investigations of patients with the disorder. It is, of course, possible that the cognitive deficits associated with HFI have in the past been merely ascribed to the cognitive decline expected to occur with normal aging, or that clinicians have been reticent to ascribe cognitive deficits to a disorder with vague neurological implications. Certainly, with people now living to advanced ages, and with the increased availability of neuroimaging techniques, cases of HFI may be more often seen and documented. We suggest that further attempts should be made to identify such cases, and the cognitive sequelae associated with HFI investigated more extensively.

We thank Graham Galloway for his assistance with imaging of control subjects, as well as Jenny Whitehead and Pauline Doddrell for their assistance with patient appointments. This study was supported by a grant from SmithKline Beecham Pharmaceuticals, UK to the Centre for Magnetic Resonance, University of Queensland.

1 Armelagos G, Chrisman OD. Hyperostosis frontalis interna: a Nubian case. Am F Phys Anthropol 1988;76:25-8.

2 Morgagni GB. Adversaria anatomica. Padua, 1719. (Francis A Coutway Library of Medicine, 1984)

3 Stewart RM. Localized cranial hyperostosis in the insane. $\mathcal{F}$ Neurol Psychopathol 1928;8:321.

4 Morel F. L'hyperostose Frontale Interne. Geneva: Chapalay and Mottier, 1929

5 Adams RD, Victor M. Principles of neurology. New York: McGraw-Hill, 1993:532.

6 Lishman WA. Organic psychiatry. 2nd ed. Oxford: Blackwell Science, 1987:653.

7 Owen AM, Evans AC, Petrides M. Evidence for a two-stage model of spatial working memory processing within the lateral frontal cortex: a positron emission tomography study. Cere Cortex 1996;6:31-8.

8 Ptudy. Cere Cortex 1996;6:31-8. from investigations of cortical excisions in nonhuman primates. In: Boller F, Grafman J, eds. Handbook of neuropsychology. Vol 9. Amsterdam: Elsevier, 1994:59-82.

9 Duncan J. Attention, intelligence and the frontal lobes. In: Gazzaniga M, ed. The cognitive neurosciences. Cambridge, MA: MIT Press, 1995:721-33.

10 Duncan J, Burgess, P, Emslie H. Fluid intelligence after frontal lobe lesions. Neuropsychologia 1995;33:261-8.

11 Owen AM, Downes JJ, Sahakian BJ, Polkey CE, Robbins TW. Planning and spatial working memory following frontal lobe lesions in man. Neuropsychologia 1990;28:1021-34. 12 Owen AM, Morris RG, Sahakian BJ, Polkey CE, Robbins, TW. Double dissociations of memory and executive fun. functions in working memory tasks following frontal lobe excisions, temporal lobe excisions or amygdalo3 Spearman C. The abilities of man. New York: Macmillan, 1927.

14 Wechsler D. Wechsler adult intelligence scale. New York: Psychological Corporation, 1955. 
15 Teuber HL. Unity and diversity of frontal lobe functions. Acta Neurobiol Exp (Warsz) 1972;32:615-56.

16 Folstein MF, Folstein SE, McHugh PR. Mini-mental state: a practical method of grading the cognitive state of patients

17 Wechsler D. Wechsler adult intelligence scale - revised. New York: Psychological Corporation, 1981

18 Nelson H, Willison J. National adult reading test. 2nd ed. Windsor, Berkshire: NFER-Nelson, 1991.

19 Warrington EK. Recognition memory test. Windsor, Berkshire: NFER-Nelson, 1984

20 Brandt J. The Hopkins verbal learning test: development of a new memory test with six equivalent forms. Clin Neuropsychol 1991;5:125-42.

21 Kaplan E, Goodglass H, Weintraub S. Boston naming test. Philadelphia: Lea and Febiger, 1983.

22 Baddeley A, Emslie H, Nimmo Smith I. The speed and capacity of language processing test. Bury St Edmunds: Vest Company, 1992.

23 Warrington EK, James M. Visual object and space perception battery. Bury St Edmunds: Thames Valley Test Company, 1991.

24 Teuber HL. The riddle of frontal lobe function in man. In Warren J, Akert K, eds. The frontal granular cortex and behavior. New York: McGraw-Hill, 1964:410-41.

25 Paus $T$. Location and function of the human frontal eye-field: a selective review. Neuropsychologia 1996;34:475483.
26 Shallice T. Specific impairments in planning. Philos Trans $R$ Soc Lond B Biol Sci 1982;298:199-209.

27 Wilson B, Alderman N, Burgess, P, Emslie, H, Evans JJ. Behavioural assessment of dysexecutive syndrome. Bury $\mathrm{St}$ Edmunds: Thames Valley Test Company, 1996.

28 Shallice T, Burgess, P. Deficits in strategy application following frontal lobe damage in man. Brain 1991;114 $727-41$

29 Shallice T, Evans E. The involvement of the frontal cortex in cognitive estimation. Cortex 1978;14:294-303.

30 Nelson $\mathrm{H}$. A modified card sorting test sensitive to frontallobe defects. Cortex 1976;12:313-24.

31 Petrides M, Milner, B. Deficits in subject-ordered tasks after frontal and temporal lobe lesions in man. Neuropsychologia 1982;20:249-62.

32 Wiegersma S, van der Scheer E, Human R. Subjective ordering, short-term memory, and the frontal lobes. Neuropsychologia 1990;28:95-8.

33 Institute for Personality and Ability Testing. Measuring Intelligence with the culture fair tests. Champaign, ILL: Institute for Personality and Ability Testing, 1973 .

34 Shallice T. From neuropsychology to mental structure. Cambridge: Cambridge University Press, 1988.

35 Burgess P. Theory and methodology in executive function Burgess $\mathrm{P}$. Theory and methodology in executive function
research. In: Rabbitt P, ed. Methodology of frontal and executive functions. Hove: Lawrence Erlbaum, 1997 (in press).

36 Baddeley A. Working memory. Oxford: Oxford University Press, 1986 